QMW-PH-97-23

\title{
GAUGINO CONDENSATION, MODULI POTENTIAL AND SUPERSYMMETRY BREAKING IN M-THEORY MODELS
}

\author{
Zygmunt Lalak $^{*}$ and Steven Thomas ${ }^{\dagger}$ \\ * Institute of Theoretical Physics \\ University of Warsaw \\ PL-00-681 Warsaw, Poland \\ $\dagger$ Department of Physics \\ Queen Mary and Westfield College \\ Mile End Road, London E1 4NS, UK
}

\begin{abstract}
We derive the explicit form, and discuss some properties of the moduli dependent effective potential arising from M-theory compactified on $M_{4} \times X \times S^{1} / Z_{2}$, when one of the boundaries supports a strongly interacting gauge sector and induces gaugino condensation. We discuss the relation between the explicit gaugino condensate and effective superpotential formulations and find interesting differences with respect to the situation known from the weakly coupled heterotic string case. The moduli dependence of the effective potential turns out to be more complicated than expected, and perhaps offers new clues to the stabilization problem.
\end{abstract}




\section{Introduction}

Lack of understanding of the mechanism of realistic supersymmetry breaking is the crucial missing ingredient in supersymmetric theories of fundamental forces and the obstinate roadblock in the supersymmetric unification programme. The other troublesome problem in these schemes is the apparent lack of unification between gauge and gravitational couplings. Although the low energy considerations suggest unification of gauge couplings in supersymmetric extensions of the Standard Model at the mass scale $M_{G U T}<M_{P L A N C K}$, the Newton constant scaled with energy as $E^{2}$ comes out to be too small to unify with the other couplings at $M_{G U T}$ in standard scenarios, cf. [1]. The way to avoid this trouble has been suggested by Witten, [2], in the framework of the field theoretical limit of the strongly coupled string theory, usually referred to as field theoretical limit of the M-theory. Witten and Horava [3] have argued that the effective low energy field theory stemming from M-theory and describing the low-energy limit of the strongly coupled heterotic string is the 11-dimensional supergravity on the manifold with boundary, which couples to 10d supersymmetric Yang-Mills theories living on the components of the 10dimensional boundary.

In the model of Witten and Horava there are two components of the boundary each containing one $E_{8}$ super-YM sector. The two sectors communicate with each other through the gravitational mediation, and, interestingly enough, in the regime where the requirement of the extended unification in the visible world is fulfilled, one of them is more strongly coupled than the other. This suggests that we are given in a natural way the much desired $4 \mathrm{~d}$ supergravity hidden sector with the strongly coupled $E_{8}$ group, where we expect gauge fermions, gauginos, to condense at the dynamically generated scale $\Lambda_{8}$. Given this observation, one can hope that this condensation can be the natural source of supersymmetry breaking in the model (in analogy with classic considerations in the weakly coupled heterotic string models, [4], [5]). Indeed, the work of Horava, [6], shows, that hidden sector gaugino condensation breaks 11d supersymmetries, and does it in a very interesting and nontrivial way. However, this phenomenon deserves further investigation.

For instance, since the visible sector is separated from the condensing sector, it is not obvious what is the specific form of the local operators violating supersymmetry in the visible sector. The hypothesis of Antoniadis and Quiros [7] (cf. also Dudas and Grojean [8]) puts forward the version of the Scherk-Schwarz mechanism as the effective description of the gaugino condensation. The Scherk-Schwarz mechanism however implies a very 
specific contribution to the potential energy density. One should be able to understand this contribution starting from the fundamental Lagrangian with multifermionic terms, which hasn't been attempted yet. Next, the picture of Horava assumes the stiff condensate, which is not a dynamical variable subject to backreaction of other dynamical degrees of freedom. In the reality this is not correct, as the condensate is going to be a function of several moduli fields, actually forms a dynamically generated potential for these fields, and its actual magnitude should be determined upon the minimization of the potential over the moduli space. This brings back the problem of moduli stabilization and its possible connection to supersymmetry breaking mechanism - it would be very disappointing if there wouldn't be any. We stress here the fact that the condensate is in fact a dynamical variable, following the classic work of Dine et. al [5], as it has important consequences in the weakly coupled heterotic string.

There it turns out that, if one forgets about T-duality, the dynamical condensate tends to adjust itself in such a way that supersymmetry remains unbroken, and moduli run away into ultra-weakly coupled regime. In those models one needs a specific T-dual superpotential for the moduli, and usually some subsidiary tools like chiral matter in the hidden sector, to get susy breaking in the direction of one of the moduli. Here, as the breaking is associated with boundary conditions which project away solutions to the Killing spinor equations, one would expect that once the condensate is nonzero, supersymmetry is broken, but still there remains the question about the magnitude of the condensate (and the gravitino mass which is related to it) and about the form of the effective potential for the moduli.

Finally, there is the associated problem of the general form of the effective Lagrangian seen at low energies in the 4-dimensional observable sector. This sector contains at least two pieces

$$
\mathcal{L}=\mathcal{L}_{\text {obs }}(Q, M)+\mathcal{L}_{\text {moduli }}(M)
$$

where $Q$ denotes observable fields and $M$ are moduli - which don't have to be much heavier than $1 \mathrm{TeV}$. It is interesting to note, that usually authors tend to think even in the context of M-theory models that this Lagrangian should be derivable from some effective $4 \mathrm{~d}$ supergravity, which then - from the $4 \mathrm{~d}$ point of view - breaks down spontaneously, and that there must exist a $4 \mathrm{~d}$ superpotential for moduli M. However, in the present context, when supersymmetry breaking in $11 \mathrm{~d}$ (or $5 \mathrm{~d}$ after obvious compactification) arises from boundary conditions in 11th (or 5th) dimension, what one can naturally expect is explicit supersymmetry in $5 \mathrm{~d}$, so for instance the existence of the effective superpotential for 
moduli is not really obvious. This seems to be an important point, as one tends to describe the supersymmetry breaking at low energies in terms of F-terms, and the nonvanishing ones are expected to arise exactly in the moduli sector. To discuss seriously and reliably the F-terms one needs the form of the effective moduli potential (and superpotential). This is the fundamental question which we raise and attempt to discuss in this paper.

To perform our task we shall used mixed techniques, reducing the fermionic terms from 10 dimensions and then deducing moduli dependence of the condensate through the gauge coupling dependence of various scales, but also trying to construct directly the effective superpotential for moduli.

Our paper is structured as follows: in section 2 we consider compactification of the Mtheory effective field theory, corresponding to strongly coupled $E_{8} \times E_{8}$ heterotic strings, in which gaugino condensation occurs on one of the two boundaries, in the presence of non vanishing $G_{11 A B C}$. The emphasis is on the computation of the moduli dependence of the resulting four dimensional effective action. In section 3 we consider to what extent such an effective potential can be reconstructed from standard $N=1, d=4$ supergravity with a moduli dependent superpotential $W(S, T)$ whose form has already been proposed in the recent literature. We shall achieve only partial success in this task, encountering a number of problems which appear, at least, to have a common origin. Some qualitative remarks concerning the possible stabilization of moduli expectation values are also made. With the various difficulties referred to above in mind, we make some preliminary comments in section 4 concerning the origin of soft supersymmetry breaking masses in potentially realistic models. We end with conclusions.

\section{Gaugino condensation and effective potential}

To start with let us recall the form of the M-theory Lagrangian constructed by Horava and Witten [3], which is given by $L_{S}+L_{B}$ where

$$
\begin{aligned}
L_{S} & =\frac{1}{\kappa^{2}} \int_{M^{11}} d^{11} x \sqrt{g}\left\{-\frac{1}{2} R-\frac{1}{2} \bar{\Psi}_{I} \Gamma^{I J K} D_{J} \Psi_{K}-\frac{1}{48} G_{I J K L} G^{I J K L}\right. \\
& -\frac{\sqrt{2}}{384}\left(\bar{\Psi}_{I} \Gamma^{I J K L M N} \Psi_{N}+12 \bar{\Psi}^{J} \Gamma^{K L} \Psi^{M}\right)\left(G_{J K L M}+\hat{G}_{J K L M}\right) \\
& \left.-\frac{\sqrt{2}}{3456} \epsilon^{I_{1} \ldots I_{11}} C_{I_{1} I_{2} I_{3}} G_{I_{4} \ldots I_{7}} G_{I_{8} \ldots I_{11}}\right)
\end{aligned}
$$




$$
\begin{aligned}
L_{B} & =\frac{1}{2 \pi\left(4 \pi \kappa^{2}\right)^{2 / 3}} \sum_{m=1}^{2} \int_{M_{m}^{10}} d^{10} x \sqrt{g}\left(-\frac{1}{4} \operatorname{Tr} F_{A B}^{m} F^{m A B}-\frac{1}{2} \operatorname{Tr} \bar{\chi}^{m} \Gamma^{A} D_{A}(\hat{\Omega}) \chi^{m}\right. \\
& \left.-\frac{1}{8} \operatorname{Tr} \bar{\Psi}_{A} \Gamma^{B C} \Gamma^{A}\left(F_{B C}^{m}+\hat{F}_{B C}^{m}\right) \chi^{m}+\frac{\sqrt{2}}{48} \operatorname{Tr} \bar{\chi}^{m} \Gamma^{A B C} \chi^{m} \hat{G}_{A B C 11}\right) \\
& +O\left(\kappa^{4 / 3}\right) \text { relative to } L_{S}
\end{aligned}
$$

where in eqs(2, 3) $, I=1, . .11$ label coordinates on $M_{11} ; A=1 . .10$ those on $M_{4} \times X$, ( $a, \bar{b}=1 . .3$ will denote the holomorphic and antiholomorphic coordinates on $X$.) The field strength $G_{I J K L}=\left[\partial_{I} C_{J K L} \pm 23\right]$ terms $+O\left(\kappa^{2}\right)$ satisfy the modified Bianchi identities [3]

$$
(d G)_{11 A B C D}=-\frac{3 \sqrt{2} \kappa^{2}}{\lambda^{2}} \sum_{m=1}^{2} \delta^{(m)}\left(x^{11}\right)\left[\operatorname{Tr} F_{[A B}^{m} F_{C D]}^{m}-\frac{1}{2} \operatorname{Tr} R_{[A B} R_{C D]}\right]
$$

and in particular one can solve (4) by defining a modified field strength [3]

$$
G_{11 A B C}=\left(\partial_{11} C_{A B C} \pm 23 \text { terms }+\frac{\kappa^{2}}{\sqrt{2} \lambda^{2}} \sum_{m=1}^{2} \delta^{(m)}\left(x^{11}\right)\left(\omega_{A B C}^{(m)}-\frac{1}{2} \omega_{A B C}^{(L)}\right)\right.
$$

where $\omega^{(m)}$, and $\omega^{(L)}$ are $\left(E_{8}\right)$ Yang Mills and Lorentz Chern-Simons 3 forms defined on the respective boundaries, and $\lambda^{2}=2 \pi\left(4 \pi \kappa^{2}\right)^{2 / 3}$ is the $d=10$ gauge coupling constant. $\kappa=m_{11}^{-9 / 2}$, with $m_{11}$ the 11 dimensional Planck mass. The delta functions $\delta^{(m)}\left(x^{11}\right)$ have support on the two fixed point sets in $M_{4} \times X \times S^{1} / Z_{2}$. The presence of these various source terms in eqs(4,5) are an important difference with the corresponding Bianchi identities relevant to compactification of the perturbative $E_{8} \times E_{8}$ heterotic string, where $H_{A B C}$ plays the role of $G_{11 A B C}$.

In the bulk $d=11$ supergravity lagrangian $L_{S}, g=\operatorname{det}\left(g_{I J}\right)$ involves the $d=11$ bulk metric. In the boundary lagrangian, the same quantity is understood as being the determinant of the $d=10$ metric obtained as the restriction of the bulk metric to either of the two boundaries $M_{m}^{10}, m=1,2$. Similarly the two copies of the $E_{8}$ super Yang Mills fields defined on the boundaries are denoted by $F^{m A B}, \chi^{m}$ respectively. $\Omega_{A B C}$ are the usual $d=10$ spin connections, with hatted quantities denoting the supercovariant generalizations, explicit definitions of which can be found in [3].

We shall be interested in compactification of the terms in $L_{S}+L_{B}$ relevant to the derivation of the effective potential $V_{\text {eff }}$ obtained when gaugino condensation occurs on one of the boundaries corresponding to the hidden sector (which we identify as the boundary component $M_{m=2}^{10}$ ) in the presence of a non vanishing $G_{11 A B C}$. In compactifying to $d=4$ 
we shall adopt a similar procedure discussed in [8] where the sequence is $11 \rightarrow 5 \rightarrow 4$ in the bulk action and $10 \rightarrow 4$ on the boundaries. The other subtlety that occurs in M-theory compactification to $d=4$ arises because the internal six dimensional metric $g_{i j}, i=4, . .9$ is a function in general of the orbifold coordinate $x^{11}$ as well as $x^{i}$. Indeed it was shown in [2] that this is a necessary condition in order to get unbroken $N=1$ supersymmetry in four dimensions. In what follows we want to consider the dependence of $V_{\text {eff }}$ on the moduli associated with the overall scales of $g_{i j}$ and the $Z_{2}$ invariant metric $g_{11,11}$. Thus in the spirit of [8] we assume we have metrics $g_{i j}^{(0)}\left(x^{11}, x^{i}\right)$ and $g_{11,11}^{(0)}$ which have the right shape but the wrong "size", i.e. we write in compactifying from $d=11 \rightarrow 5 \rightarrow 4$

$$
\begin{aligned}
g_{\mu^{\prime} \nu^{\prime}}^{(11)} & =e^{-2 \sigma\left(x^{\mu}\right)} g_{\mu^{\prime} \nu^{\prime}}^{(5)}, \quad g_{i j}^{(11)}=\frac{1}{2} e^{\sigma\left(x^{\mu}\right)} g_{i j}^{(0)}\left(x^{11}, x^{i}\right), \\
g_{11,11}^{(5)} & =e^{2 \gamma\left(x^{\mu}\right)} g_{11,11}^{(0)}, \quad g_{\mu \nu}^{(5)}=e^{-\gamma\left(x^{\mu}\right)} g_{\mu \nu}^{(4)}
\end{aligned}
$$

where $\mu^{\prime}=0, . .3,11 ; \mu=0 \ldots 3$ and the superscripts on the metrics in () brackets indicate the particular dimension the metric is defined in. We have emphasised in (6) that the fields $\gamma$ and $\sigma$ only depend on the four dimensional coordinates $x^{\mu}$ but each of the metrics $g_{i j}^{(0)}, g_{11,11}^{(0)}$ and $g_{\mu \nu}^{(4)}$ can depend on $x^{i}, x^{11}, x^{\mu}$ in a way determined by the requirement of unbroken $N=1, d=4$ supersymmetry [2] . Our approach thus follows more closely the original one by the authors of [5] rather than the dimensional truncation approach to $\mathrm{N}=1, \mathrm{~d}=4$ supersymmetry as employed recently in [8] [9] .

The choice of factors of $\sigma$ and $\gamma$ in (6) yields canonically normalized Einstein-Hilbert actions in $d=4$ in the supergravity basis [8], (i.e. there is a kinetic energy for the field $\gamma$ ) .

The (curved space) $\mathrm{d}=11$ gamma matrices satisfy $\left\{\Gamma^{I}, \Gamma^{J}\right\}=2 g^{(11) I J}$, and corresponding to the scalings in (6) we have the following $\sigma$ and $\gamma$ dependence of their various components after compactification :

$$
\begin{gathered}
\Gamma_{(11)}^{\mu^{\prime}}=\Gamma_{(5)}^{\mu^{\prime}} e^{\sigma}, \quad \Gamma_{(11)}^{i}=\Gamma_{(5)}^{i} e^{-\sigma / 2} \\
\left\{\Gamma_{(5)}^{\mu^{\prime}}, \Gamma_{(5)}^{\nu^{\prime}}\right\}=2 g^{(5) \mu^{\prime} \nu^{\prime}}, \quad\left\{\Gamma_{(5)}^{i}, \Gamma_{(5)}^{j}\right\}=2 g^{(0) i j}, \quad\left\{\Gamma_{(5)}^{\mu^{\prime}}, \Gamma_{(5)}^{i}\right\}=0
\end{gathered}
$$

and furthermore

$$
\Gamma_{(5)}^{\mu^{\prime}=11}=\Gamma_{(4)}^{\mu^{\prime}=11} e^{-\gamma}, \quad \Gamma_{(5)}^{\mu}=\Gamma_{(4)}^{\mu} e^{\gamma / 2}
$$




$$
\left\{\Gamma_{(4)}^{\mu}, \Gamma_{(4)}^{\nu}\right\}=2 g^{(4) \mu \nu}, \quad\left\{\Gamma_{(4)}^{11}, \Gamma_{(4)}^{11}\right\}=2 g^{(0) 11,11}, \quad\left\{\Gamma_{(4)}^{\mu}, \Gamma_{(4)}^{11}\right\}=0
$$

Horava has shown that the combination of terms involving $G_{11 A B C}$ and $\chi$ in $L_{S}$ and $L_{B}$ can be written as a bulk perfect square action $L_{s q}$ :

$$
L_{s q}=-\frac{1}{12 \kappa^{2}} \int_{M^{11}} d^{11} x \sqrt{g}\left(G_{11 A B C}-\frac{\sqrt{2}}{16 \pi}\left(\frac{\kappa}{4 \pi}\right)^{2 / 3} \delta^{(2)}\left(x^{11}\right) \operatorname{Tr} \bar{\chi} \Gamma_{A B C} \chi\right)^{2}
$$

where it should be emphasised that the perfect square term should transform as a scalar with respect to $d=11$ coordinate transformations hence a factor of $g^{11,11}$ is implicit in (9). (Recall that the delta functions $\delta^{(m)}\left(x^{11}\right)$ are not invariant, but rather transform as covariant vectors under coordinate transformations of $x^{11}$ ). Integrating (9) over $x^{11}$ we have:

$$
\begin{aligned}
L_{s q}= & -\frac{1}{12 \kappa^{2}} \int_{M^{11}} d^{11} x \sqrt{g_{(11)}}\left(G_{11 A B C} G_{11}^{A B C} g^{11,11}\right) \\
& +\frac{1}{6 \kappa^{2}} \frac{\sqrt{2}}{16 \pi}\left(\frac{\kappa}{4 \pi}\right)^{2 / 3} \int_{M_{2}^{10}} d^{10} x \sqrt{g_{(10)}} \operatorname{Tr}\left(G_{11 A B C} \bar{\chi} \Gamma^{A B C} \chi\right)\left(\sqrt{g_{11,11}}\right)^{-1} \\
& -\frac{1}{12 \kappa^{2}}\left(\frac{\sqrt{2}}{16 \pi}\left(\frac{\kappa}{4 \pi}\right)^{2 / 3}\right)^{2} \int_{M_{2}^{10}} d^{10} x \sqrt{g_{(10)}} \operatorname{Tr}\left(\bar{\chi} \Gamma_{A B C} \chi\right)\left(\bar{\chi} \Gamma^{A B C} \chi\right) \delta^{i n v}(0)
\end{aligned}
$$

where $\delta^{(i n v)}\left(x^{11}\right) \equiv\left(\sqrt{g_{11,11}}\right)^{-1} \delta\left(x^{11}\right)$ is a delta function transforming as a scalar under $x^{11}$ coordinate transformations.

An important feature of M-theory compactification as shown by Witten [2], is the fact that the volume associated with the compact six dimensional internal spaces at each of the boundaries $M_{i}^{10}=M^{4} \times X_{i}$ are different. The interpolating metrics $g_{i j}\left(x^{i}, x^{11}\right)$ in (6) depend on $x^{11}$ in general, and in [2] it was shown, by considering an expansion about an $x^{11}$ independent metric, that the volumes $V_{m}$ of $X_{m}, m=1,2$ defined at the two fixed point sets $x^{11}=0$ and $x^{11}=\pi \rho$ are related:

$$
\begin{aligned}
V_{2}(\sigma, \gamma) & =V_{1}(\sigma, \gamma)-\frac{\pi}{4 \sqrt{2}} \rho \int_{X_{2}} d^{6} x \sqrt{g} \alpha(g, F) \\
& =V_{1}(\sigma, \gamma)-\frac{\pi}{4 \sqrt{2}} e^{\gamma} m_{11}^{-1} \int_{X_{2}} d^{6} x \sqrt{g^{(0)}} \alpha\left(g^{(0)}, F\right)
\end{aligned}
$$

where $d^{6} x \sqrt{g} \alpha(g, F)=\frac{\sqrt{2}}{\pi}\left(\frac{\kappa}{4 \pi}\right)^{2 / 3} \omega \wedge\left[\operatorname{Tr} F^{(2)} \wedge F^{(2)}-\frac{1}{2} \operatorname{Tr} R \wedge R\right]$ is a 6-form defined wrt the metric $g_{i j}$, ( $\omega$ being the Kähler form ), and $\rho$ is the length of the line element $S^{1} / Z_{2}$ with 
respect to the metric choice $g_{11,11}=1$ [2]. In obtaining the second line in (11]), we have used the $\sigma$ and $\gamma$ dependence given in (6) and the relation $\rho=e^{\gamma-\sigma} m_{11}^{-1}$, which expresses the radius in supergravity units.

The volumes $V_{1}(\sigma, \gamma)$ and $V_{2}(\sigma, \gamma)$ define the four dimensional observable and hidden sector gauge coupling constants $g_{w}$ and $g_{h}$, 田

$$
\frac{1}{g_{w}^{2}}=\frac{8 \pi V_{1}}{\left(4 \pi \kappa^{2}\right)^{2 / 3}} \quad, \quad \frac{1}{g_{h}^{2}}=\frac{8 \pi V_{2}}{\left(4 \pi \kappa^{2}\right)^{2 / 3}}
$$

The result found by Witten (11) translates directly into the statement that the difference $g_{h}^{-2}-g_{w}^{-2}$ depends only on the particular integral defined in (11). Another way of understanding this result, from a weak coupling perspective was presented by Banks and Dine [10]. They pointed out that if the effective four dimensional gauge couplings are small and one considers a large radius Calabi-Yau space, the individual moduli dependence of $g_{w}$ and $g_{h}$ can be computed. The result follows directly as a consequence of supersymmetry and holomorphicity once the axionic couplings are known. Such couplings can be determined from reducing the $d=10$ Green-Schwarz terms. For a single overall $(1,1)$ modulus $T$ and modulus $S$ of the weakly coupled 10 dimensional theory, the couplings are given by

$$
\begin{aligned}
g_{w}^{-2} & =(32 \pi)^{1 / 3}(\operatorname{Re} S)+\frac{m_{11}^{2}}{4(4 \pi)^{1 / 3}}(\operatorname{Re} T) \int_{X_{2}} d^{6} x \omega \wedge\left[\operatorname{Tr} F^{(1)} \wedge F^{(1)}-\frac{1}{2} \operatorname{Tr} R \wedge R\right] \\
g_{h}^{-2} & =(32 \pi)^{1 / 3}(\operatorname{Re} S)-\frac{m_{11}^{2}}{4(4 \pi)^{1 / 3}}(\operatorname{Re} T) \int_{X_{2}} d^{6} x \omega \wedge\left[\operatorname{Tr} F^{(2)} \wedge F^{(2)}-\frac{1}{2} \operatorname{Tr} R \wedge R\right](13)
\end{aligned}
$$

where we emphasise that here $S$ is the weak coupling modulus, whose real part is related to the dilaton in the usual way.

The question arises whether (13) might be true even if one is in a region where the M-theory description is more appropriate. The answer appears to be affirmative, because it has been noted in [3] that the Chern-Simons term $C G G$ in the action (2) turns into an effective Green-Schwarz term, when one uses the relation

$$
G_{A B C D}=-\frac{3 \kappa^{2}}{\sqrt{2} \lambda^{2}} \epsilon\left(x^{11}\right)\left(\operatorname{Tr} F_{[A B} F_{C D]}+\ldots \ldots\right)
$$

which solves the modified Bianchi identity (四) near $x^{11}=0$. Taking into account both boundaries, one could once again derive axionic couplings following the arguments of [10]

\footnotetext{
${ }^{1}$ With the standard embedding of the spin connection into the gauge connection occuring at the boundary $X_{1}$ the group $E_{8}$ is broken to $E_{6}$ and the corresponding coupling $g_{w}$ is smaller than $g_{h}$ associated with the boundary $X_{2}$.
} 
and hence from supersymmetry and holomorphicity, conclude that the tree level moduli dependence of $g_{w}$ and $g_{h}$ has the same functional form as in (13) but with $S$ and $T$ moduli given by

$$
S=e^{3 \sigma}+i \theta_{1}, \quad T=e^{\gamma}+i \theta_{2}
$$

$\theta_{1}$ and $\theta_{2}$ being related to the axionic degrees of freedom.2

The the idea that certain features of the perturbative gauge coupling threshold corrections can be successfully extrapolated to strong coupling has been discussed in [14], based on anomaly cancellation arguments. The form of the couplings in (13) is clearly a manifestaion of this and has been used recently in the discussion of the pattern of the soft supersymmetry breaking masses in M-theory [15.

In studying the mechanism of gaugino condensation, we shall assume that the boundary $X_{2}$ has non contractible cycles which allow one to turn on Wilson lines corresponding to the the hidden sector $E_{8}$, breaking the latter to some group $G$ whose coupling constant becomes strong at some scale $\mu$. We want to determine the dependence of $\mu$ on $\gamma$ and $\sigma$. General arguments relate the scale $\mu$ to the GUT scale $M_{G U T}$ which is in turn given by the masses of the gauge mesons corresponding to the broken generators in the process $E_{8} \rightarrow G$.

Considering the compactification of the pure Yang-Mills action on $M_{2}^{10}$ to $d=4$ the relevant terms are

$$
S_{Y M}^{(4)}=-\frac{1}{4}\left(\frac{1}{4 \pi g_{h}{ }^{2}}\right) \int d^{4} x\left(\operatorname{Tr} F_{\mu \nu} F^{\mu \nu}+e^{-3 \sigma-\gamma} \operatorname{Tr} D_{\mu} A_{i} D^{\mu} A^{i}+\ldots\right)
$$

Wilson lines correspond to the scalars $A_{i}$ transforming in the adjoint of $E_{8}$ acquiring non vanishing expectation values. The scale of $\left\langle A^{i}\right\rangle$ can be determined by the requirement that metric independent integral $\int_{\Gamma}\langle A\rangle \cdot d \mathbf{x}$ (where $\Gamma$ is a non-contractible cycle in $X_{2}$ ) is of order unity. This then implies that $\langle A\rangle$ is typically of order $m^{-1}{ }_{11}$. To determine the gauge meson masses, we have to rescale the $d=4$ fields $A_{\mu}$ and $A_{i}$ appearing in (16) to obtain canonical kinetic energies. Thus we define the canonical fields $\tilde{A}_{\mu}, \tilde{A}_{i}$

$$
A_{\mu}=2 \sqrt{\pi} g_{h} \tilde{A}_{\mu}, \quad A_{i}=2 \sqrt{\pi} g_{h} e^{3 \sigma / 2+\gamma / 2} \tilde{A}_{i}
$$

from which it follows that,

$$
M_{G U T} \approx M_{\text {meson }} \approx m_{11} e^{-3 \sigma / 2-\gamma / 2}, \quad \mu(\sigma, \gamma) \approx M_{G U T} e^{-1 / 2 b_{0} g_{h}^{2}(\sigma, \gamma)}
$$

\footnotetext{
${ }^{2}$ These general conclusions have also been found in a recent analysis of the four dimensional effective action obtained from the Horava-Witten Lagrangian [16], where the authors include additional effects due to low energy gauge fields.
} 
where $b_{0}$ is the coefficient of the first term in the beta function associated with the coupling $g_{h}$, and $\mu$ is defined as the scale at which the running coupling $g_{h}$ becomes strong. In the perturbative case [5], there is a similar expression for $\mu$ except there the multiplicative factor is $e^{-2 \sigma}$ only, and the M-theory mass scale $m_{11}$ is replaced by the $d=4$ Planck mass $M_{p l}$.

In order to discuss hidden sector gaugino condensation, we need also to define canonical kinetic terms for the $d=4$ gauginos associated with the gauge group $G$ of the strongly coupled sector. Here by canonical we mean that the gauginos are normalized in the same way as the corresponding gauge fields, as required by unbroken $N=1$ supersymmetry. Using the metric ansatz of (6), and the various rescalings of the gamma matrices given in (78.8) the kinetic energy for the massless $d=4$ gauginos of the group $G$ are

$$
\int d^{4} x\left\{-\frac{1}{2 g_{h}{ }^{2}} e^{-2 \sigma-\gamma} \operatorname{Tr} \bar{\chi} \gamma^{\mu} D_{\mu} \chi\right\}
$$

from which we learn that the correctly normalized four dimensional gaugino $\chi_{(4)}=$ $\sqrt{2} e^{-\sigma-\gamma / 2} \chi$. With this normalization we expect that the strongly interacting gauge group $G$ induces nonvanishing expectation values for gaugino bilinears at a mass scale determined by $\mu(\sigma, \gamma)$ i.e. $\left|\left\langle\bar{\chi}_{(4)} \chi_{(4)}\right\rangle\right| \sim \mu^{3}(\sigma, \gamma)$.

In principle, to obtain the supersymmetric Wilsonian action below the scale $\mu$ one should integrate correctly all massive modes from $m_{11}$ down to $\mu$ in a supersymmetry preserving way. Assuming this can be done, one ends up with a supersymmetric effective action. Here we adopt a practical approach and employ the simplified procedure of Dine et al. [5].

Now we want to derive an effective potential from the terms in (10) whose origin was the perfect square term in (9). The procedure we shall adopt is to replace gaugino bilinears in terms of the quantity $\mu^{3}(\sigma, \gamma)$ allowing at the same time complex phases in the latter. Whether the perfect square structure, which is apparent in $d=11$ survives compactification would seem to depend on the precise form we take for $G_{11 i j k}$ and the bilinears $\left\langle\bar{\chi} \Gamma_{i j k} \chi>\right.$. The situation appears more complicated than in the perturbative case. There for example instead of $G_{11 i j k}$ we have the 3 form $H=d B+\omega^{Y M}-\omega^{L}$, the latter terms being the difference between Yang- Mills $\left(E_{8} \times E_{8}\right)$ and Lorentz ChernSimons 3-forms. Although the possibility of turning on these latter terms was raised in [5], the usual procedure is to allow dirac like string singularities in the potential $B_{i j}$ such that $H_{i j k}=c \epsilon_{i j k}$, where $c$ is a constant. (Here we momentarily change our notaion so that $i$ refers to holomorphic internal indices). Then the 3-form $H$ is closed. This is consistent 
with the Bianchi identities if the topological condition $\int \operatorname{Tr} F \wedge F-\operatorname{Tr} R \wedge R=0$ holds. This constraint can be achieved by the usual embedding of the spin connection in the gauge connection, which also implies the vanishing of the Chern-Simons terms in $H_{i j k}$. At the same time general arguments lead one to take $\left\langle\bar{\chi} \Gamma_{i j k} \chi>\right.$ to also be proportional to the components $\epsilon_{i j k}$ of the holomorphic 3 form on the internal Calabi-Yau manifold. (This follows because massless $\mathrm{d}=4$ gauginos in this case, are associated with spinors on the Calabi-Yau space that are singlets under the $S U(3)$ holonomy group). The result of this is that the perfect square structure of the potential persists in $d=4$.

In the case of M-theory, $G_{11 A B C}$ as given in (5), is analogous to $H_{A B C}$ in the perturbative case, except that the Chern-Simons term has been "split apart", and has support only at the $Z_{2}$ fixed points sets. The importance of this as pointed out in [2] , is that while the standard embedding is sufficient to allow a solution to the $(d=11)$ Bianchi identities, (since this involves integrating the right hand side of (4), which picks up both fixed point contributions ), it is not sufficient for pointwise cancellation of the Yang-Mills and Lorentz Chern-Simons terms in $G_{11 A B C}$. As has been suggested [2], this might imply that $G_{11 A B C} \neq 0$ generically in M-theory compactifications.

Thus it would appear that the Chern-Simons source terms in $G_{11 A B C}$ are something one should take into consideration when calculating the effective action. Additionally, one could of course consider turning on the $C_{11 i j}, C_{i j k}$ potentials, and to this extent the perfect square form of the potential written as a $d=11$ integral (5) suggests that the latter potentials are such that $G_{11 i j k}$ develops a vev localized on the strongly coupled boundary, to compensate the similarly localized gaugino condensate [6]. We can in principle take into account both these possibilities by taking $G_{11 i j k}=\sum_{m=1}^{2} \omega_{i j k}^{(m)}\left(x^{i}\right) \delta^{(m)}\left(x^{11}\right)$, where $\omega_{i j k}^{(m)}$ could represent either the Chern-Simons terms or taking $\omega_{a b c}^{(1)}=0, \omega_{a b c}^{(2)}=c m_{11}^{3} \epsilon_{a b c}$ we would get an expression similar to the perturbative case.

Similarly, the issue arises as to the form of the fermion bilinear $\left\langle\bar{\chi} \Gamma_{i j k} \chi\right\rangle$. For now we shall be equally general and define $\operatorname{Tr}\left\langle\bar{\chi} \Gamma_{i j k} \chi\right\rangle=\mu^{3} \tilde{\omega}_{i j k}\left(x^{i}\right)$ describing a condensate localized only on the boundary $X_{2}$.

With these ansatzes the naive effective potential obtained from (10) can be computed. Before we do this, the obvious difficulties associated with the presence of singular terms involving $\delta(0)$ have to be considered. These issues were raised in [3] where their contributions to the $d=11$ supergravity action with boundaries was first obtained. In general it is believed that these are artifacts of the singular nature of the boundary and its purely classical description. Quantum M-theory effects may serve to regularize such singularities by thickening the boundaries, a typical length scale being the M-theory scale $m_{11}^{-1}$. In 
what follows we shall adopt this approach, and replace the invariant delta function $\delta^{i n v}(0)$ with $a m_{11}^{-1}$, where the arbitrary constant $a$ serves simply to rescale the effective action in $d=4$. Hence for simplicity we set $a=1$.

Taking into account the various normalizations and scalings discussed earlier, we arrive at the following form for the effective potential:

$$
\begin{aligned}
V_{\text {eff }}(\sigma, \gamma) & =\frac{m_{11}^{4}}{96 \pi^{2}(4 \pi)^{4 / 3}} e^{-3 \sigma-3 \gamma}\left\{B_{1}+B_{2}+2 B_{3} \mu^{3}(\sigma, \gamma) e^{9 \sigma / 2+3 \gamma / 2}\right. \\
& \left.+B_{4} \mu^{6}(\sigma, \gamma) e^{9 \sigma+3 \gamma}\right\}
\end{aligned}
$$

where the quantities $B_{1} \ldots B_{4}$ are given by the following integrals

$$
\begin{aligned}
& B_{1}=\int_{X_{1}} d^{6} x \sqrt{g^{(0)}} \omega_{i j k}^{(1)} \omega_{i^{\prime} j^{\prime} k^{\prime}}^{(1)} g^{(0) i i^{\prime}} g^{(0) j j^{\prime}} g^{(0) k k^{\prime}} \\
& B_{2}=\int_{X_{2}} d^{6} x \sqrt{g^{(0)}} \omega_{i j k}^{(2)} \omega_{i^{\prime} j^{\prime} k^{\prime}}^{(2)} g^{(0) i i^{\prime}} g^{(0) j j^{\prime}} g^{(0) k k^{\prime}} \\
& B_{3}=\int_{X_{2}} d^{6} x \sqrt{g^{(0)}} \omega_{i j k}^{(2)} \tilde{\omega}_{i^{\prime} j^{\prime} k^{\prime}} g^{(0) i i^{\prime}} g^{(0) j j^{\prime}} g^{(0) k k^{\prime}} \\
& B_{4}=\int_{X_{2}} d^{6} x \sqrt{g^{(0)}} \tilde{\omega}_{i j k} \tilde{\omega}_{i^{\prime} j^{\prime} k^{\prime}} g^{(0) i i^{\prime}} g^{(0) j j^{\prime}} g^{(0) k k^{\prime}}
\end{aligned}
$$

In the above integrals, the scaling factors of $e^{\sigma}$ have been taken out, and it is understood that the metrics $g_{i j}^{(0)}$ are those obtained from the bulk metrics restricted to the appropriate boundary.

Similar integrals arise in the perturbative case, the difference being that there is a single Calabi-Yau space $K$ and a single metric (so for example $B_{1}$ is absent and $K$ can be identified with $X_{2}$. In that case the 3 forms entering the integrals are all proportional to the holomorphic 3 -form on $K[5]$, and hence the integrals $B_{2} . . B_{4}$ are all proportional to each other with coefficients that reproduce a perfect square structure in $V_{\text {eff }}$.

In the present case, there are further subtleties. This is because $B_{2} . . B_{4}$ involve integrals over the boundary component $X_{2}$ located at the fixed point set $x^{11}=\pi \rho$ whilst $B_{1}$ is defined with respect to $X_{1}$. In principle there could be a hidden dependence in $B_{2} \ldots B_{4}$ on the moduli $\sigma, \gamma$ analogous to the moduli dependence of the volume integral at $X_{2}$. One can try and determine this dependence using similar methods as in [2]. In doing this one can define the integrals $B_{2}\left(x^{11}\right), \ldots B_{4}\left(x^{11}\right)$ at an arbitrary value of $x^{11}$, and obtain a differential equations for them by taking $g_{a \bar{b}}^{(0)}\left(x^{11}, x^{i}\right)=g_{a \bar{b}}^{(0)}\left(x^{i}\right)+h_{a \bar{b}}^{(0)}\left(x^{i}, x^{11}\right)+\ldots$. 
(It should be noted that here the perturbation in the metric is $e^{-\sigma}$ times the quantity $h_{a \bar{b}}\left(x^{i}, x^{11}\right)$ defined in [2] .)

In deforming the integrals away from the boundary $X_{2}$ the only dependence on $x^{11}$ occurs through the various metric factors, since the ansatz for $\omega^{(m)}$, and $\tilde{\omega}$ implies these are $x^{11}$ independent.

Using the expressions for $\partial_{11} h_{a \bar{b}}$ derived in [2] one can obtain the following equations satisfied by $B_{2} \ldots B_{4}$ away from $X_{2}$

$$
\begin{aligned}
\partial_{11} B_{2}\left(x^{11}\right) & =\frac{3}{4 \sqrt{2}} e^{\sigma} \int_{X} d^{6} x \sqrt{g^{(0)}} \omega_{i j k}^{(2)} \omega_{i^{\prime} j^{\prime} k^{\prime}}^{(2)} g^{(0) i i^{\prime}} g^{(0) j j^{\prime}} g^{(0) k k^{\prime}} \alpha\left(g^{(0)}, F\right) \\
\partial_{11} B_{3}\left(x^{11}\right) & =\frac{3}{4 \sqrt{2}} e^{\sigma} \int_{X} d^{6} x \sqrt{g^{(0)}} \omega_{i j k}^{(2)} \tilde{\omega}_{i^{\prime} j^{\prime} k^{\prime}} g^{(0) i i^{\prime}} g^{(0) j j^{\prime}} g^{(0) k k^{\prime}} \alpha\left(g^{(0)}, F\right) \\
\partial_{11} B_{4}\left(x^{11}\right) & =\frac{3}{4 \sqrt{2}} e^{\sigma} \int_{X} d^{6} x \sqrt{g^{(0)}} \tilde{\omega}_{i j k} \tilde{\omega}_{i^{\prime} j^{\prime} k^{\prime}} g^{(0) i i^{\prime}} g^{(0) j j^{\prime}} g^{(0) k k^{\prime}} \alpha\left(g^{(0)}, F\right)
\end{aligned}
$$

Next we can derive expressions for $\partial_{11}^{2} B_{\alpha}\left(x^{11}\right), \alpha=1 . .4$. This will involve $\partial_{11} \alpha$ an expression for which can be determined through the Bianchi identities of $G_{11 A B C}$ without the source terms [3], with the result that it is a total derivative wrt $x^{i}$. In fact $\partial_{11} \alpha \sim$ $\omega^{i j} \omega^{k l}(d G)_{11 i j k l}$, where $(d G)_{11 i j k l}$ only involves derivatives wrt the coordinates $x^{i}$, and again it should be stressed that here $G_{11 i j k}$ contains no source terms, unlike that defined in (4). In the context of gaugino condensation, if such terms are turned on at all they are given in terms of the holomorphic (and antiholomorphic) 3-forms, which are covariantly constant and hence in this specific case, $\alpha$ is $x^{11}$ independent. Consequently, $\partial_{11}^{2} B_{2}\left(x^{11}\right)=$ $\partial_{11}^{2} B_{3}\left(x^{11}\right)=\partial_{11}^{2} B_{4}\left(x^{11}\right)=0$, i.e. the integrals defined in $(22)$ are $x^{11}$ independent to this order. This situation is now similar to the volume calculation of $X$, and one has the following expressions for the original integrals (21)

$$
\begin{aligned}
& B_{2}=B_{1}-\frac{3}{4 \sqrt{2}} \pi \rho e^{\sigma}\left(\int_{X_{2}} d^{6} x \sqrt{g^{(0)}}\left(\omega^{(2)}\right)^{2} \alpha\left(g^{(0)}\right)\right) \\
& B_{3}=B_{3}^{(1)}-\frac{3}{4 \sqrt{2}} \pi \rho e^{\sigma}\left(\int_{X_{2}} d^{6} x \sqrt{g^{(0)}} \omega^{(2)} \cdot \tilde{\omega} \alpha\left(g^{(0)}\right)\right) \\
& B_{4}=B_{4}^{(1)}-\frac{3}{4 \sqrt{2}} \pi \rho e^{\sigma}\left(\int_{X_{2}} d^{6} x \sqrt{g^{(0)}}(\tilde{\omega})^{2} \alpha\left(g^{(0)}\right)\right)
\end{aligned}
$$

where in (23), the integrals $B_{3}^{(1)}$ and $B_{4}^{(1)}$ are defined at the fixed point set boundary $X_{1}$, and so together with $B_{1}$ are independent of the moduli $\sigma, \gamma$. Indeed this also applies 
to the integrals over $X_{2}$ in (23), the only dependence on the moduli being through the combination $\rho e^{\sigma}$, which from our previous definitions is proportional to $e^{\gamma}$.

At this point we can see that the integrals $B_{2} \ldots . B_{4}$ have a dependence on $\gamma$ that is not present in the perturbative case. (The coefficients in perturbative case would correspond to just keeping integrals $B_{2}, B_{3}$ and $B_{4}$ and keeping just the first terms on the right hand side of (23), and identifying $X_{1}$ with the space $K$ in [5] endowed with metric $g_{i j}^{(0)}$, and setting $\omega_{a b c}^{(2)}, \tilde{\omega}$ proportional to the holomorphic 3 form $\epsilon_{a b c}$ on $K$. This reproduces the perfect square potential.)

It is hard to see how this perfect square structure holds if, as we discussed earlier, we really take into account the source terms which would appear to be present in $G_{11 a b c}$ at $x^{11}=0$ and $x^{11}=\pi \rho$, because then if $\tilde{\omega}_{a b c}$ was taken to be proportional to $\epsilon_{a b c}, B_{2}, B_{3}$ and $B_{4}$ are not obviously related to each other. If however we somehow ignore these source terms, and $G_{11 a b c}$ develops a piece purely at the boundary $X_{2}$ as advocated in [6], and again proportional to $\epsilon_{a b c}$, then the square structure survives. However, even if this is so the presence of moduli dependent terms in $B_{2} \ldots B_{4}$ may not be consistent with the existence of a superpotential. We shall discuss these issues in the next section.

\section{Effective superpotential in M-theory gaugino con- densation}

In this section we shall, following the reasoning used in the perturbative approach [5], attempt to identify an $N=1, d=4$ superpotential $W(\sigma, \gamma)$ which can reproduce, in some approximation, the above scalar potential. Before we do this we have to identify the $d=4$ Kähler structure that emerges in our compactification i.e. define complex moduli fields, whose real parts will be related to $\sigma, \gamma$ and their corresponding Kähler potential. This has been studied by various authors [8], [9] in the dimensional truncation approach, but is equally applicable in our case (just as the identification of moduli, and Kähler potential in the perturbative case [0] was carried out in more general context than dimensional truncation).

The Kähler potential of the $S$ and $T$ moduli defined earlier in (15) is 8]

$$
K=-\ln (S+\bar{S})-3 \ln (T+\bar{T})
$$

Now in order to extract a superpotential we have to be careful in considering what the effective $d=4$ Planck mass is after compactification as this enters the well known formula 
for the scalar potential in $N=1, d=4$ supergravity.

We have followed the conventions in [8] regarding the various $\sigma$ and $\gamma$ dependent scalings in the metric. After compactification one obtains an $N=1, d=4$ supergravity in the 'supergravity basis' where the $\gamma$ field has a non-vanishing kinetic energy, and the $d=4$ Einstein action is canonically normalized (i.e. all factors of $e^{\gamma}$ and $e^{\sigma}$ cancel in front of the $d=4$ curvature term.). In these units the effective four dimensional Planck mass is of order $m_{11}$, and is the basis for which the usual text book formulae for the effective potential is written (see [10 for a discussion of this point.) This is to be contrasted with the theory as expressed in M-theory units, in which $\gamma$ has no kinetic term and there is a reduced Planck mass $m_{p l}^{2}=t m_{11}^{2}$ [8].

With these points in mind, we take the following form for the superpotential $W(S, T)$

$$
\begin{aligned}
W(S, T) & =m_{11}^{3}\left\{c_{1}+c_{2} e^{-\frac{3(4 \pi)^{1 / 3}}{b_{0}}\left(S+c_{3} T\right)}\right\} \\
& =m_{11}^{3} c_{1}+\tilde{W}(S, T)
\end{aligned}
$$

where $c_{1}, c_{2}$ are complex coefficients which we want to determine by trying to match the potential coming from (25) with the one obtained from compactification in (20). The form of the exponent in (25) is obtained by expressing the hidden sector coupling constant $g_{h}$ in terms of $S$ and $T$ as given in (13), and from which the constant $c_{3}$ can easily be deduced.

Superpotentials of the form (25) have already been advocated as being relevant to gaugino condensation in M-theory 10 their general forms following from requirement of holomorphicity and shift invariance. The new twist in the expression for $W(S, T)$, compared to the perturbative case, is the presence of the $T$ modulus. Clearly this will give rise to new terms in the scalar potential compared to that case.

Even before we discuss the connection between the scalar potential arising from (25) and $V_{\text {eff }}$, there are other potential difficulties associated with the superpotential. Recall the way the expectation value of gaugino bilinears $\left\langle\lambda^{a} \lambda^{b}\right\rangle$ enters the scalar potential of the $4 \mathrm{~d}$ supergravity [11]. (In this discussion we adopt the notation where $\lambda^{a}$ denotes the components of the gaugino, $i$ labels complex moduli fields, and $a$ is an adjoint group index.) With canonically normalized (in 4d) gravitational part and gauge and gaugino kinetic terms the relevant part of the Lagrangian is

$$
V=e^{K} g^{i \bar{j}}\left(D_{i} W+\frac{1}{4} e^{-K / 2} \partial_{i} f_{a b}\left\langle\lambda^{a} \lambda^{b}\right\rangle\right)\left(D_{\bar{j}} W+\frac{1}{4} e^{-K / 2} \partial_{\bar{j}} f_{a b}\left\langle\bar{\lambda}^{a} \bar{\lambda}^{b}\right\rangle\right)+\ldots
$$

where $g^{i \bar{j}}$ is the inverse Kähler metric and rest of the notation is standard (see [11]). 
Now, let us assume there is no perturbative superpotential and switching on condensates is equivalent to switching on the nonperturbative superpotential. Then one should require

$$
D_{i} W_{n p}=\frac{1}{4} e^{-K} \partial_{i} f_{a b}\left\langle\lambda^{a} \lambda^{b}\right\rangle
$$

However, in the present case the tree-level gauge kinetic function $f_{a b}$ depends on both superfields $S$ and $T$. Hence, without specifying the exact form of $W_{n p}$ one can obtain from (27) the relation (assuming that $\left\langle\lambda^{a} \lambda^{b}\right\rangle$ is non vanishing on the boundary $X_{2}$ only, with $f$ defined to be the $E_{8}$ gauge kinetic function)

$$
\frac{\partial_{S} \log W_{n p}-\frac{1}{S+S}}{\partial_{T} \log W_{n p}-\frac{3}{T+T}}=\frac{\partial_{S} f}{\partial_{T} f}
$$

The relation (28) is incompatible with assumed holomorphicity of $W$ and $f$. The way to understand this relation in the context of Horava-Witten model is to recall that the dependence of the gauge coupling on $T$ comes in from the terms which, as pointed out in [2], can be seen as the perturbation of the Lagrangian defined on the boundary with the weakly coupled $E_{8}$. This reasoning would imply that we are not allowed to write the troublesome relation (28) as it compares terms which arise in different order of perturbative expansion. However, the puzzle is still around, as the same corrections tell us what is the difference between gauge couplings at both boundaries, and obviously enter the expression for the hidden $E_{8}$ condensation scale, and, as a consequence, any expression for $W_{n p}$ we can arrive at. Of course, this particular problem with holomorphicity doesn't imply on its own that there is something wrong with supersymmetry - even standard $4 \mathrm{~d}$ supergravity with loop corrections in general cannot be formulated in the canonical form known from standard tree level formulation. In what follows we shall encounter further trouble with holomorphicity, arising specifically in M-theory model we have here.

Returning to the calculation of the scalar potential, $W(S, T)$ together with the Kähler potential $K(S, T)$ given in (24) gives the following

$$
\begin{aligned}
V_{\text {eff }} & =m_{11}^{4} e^{-3 \sigma-3 \gamma}\left\{e^{6 \sigma}\left|\left(e^{-3 \sigma} \frac{W(S, T)}{m_{11}^{3}}+6 c_{2} \frac{(4 \pi)^{1 / 3}}{b_{0}} e^{-\frac{3(4 \pi)^{1 / 3}}{b_{0}}\left(S+c_{3} T\right)}\right)\right|^{2}\right. \\
& +3 e^{2 \gamma}\left|\left(e^{-\gamma} \frac{W(S, T)}{m_{11}^{3}}+6 c_{2} c_{3} \frac{(4 \pi)^{1 / 3}}{b_{0}} e^{-\frac{3(4 \pi)^{1 / 3}}{b_{0}}\left(S+c_{3} T\right)}\right)\right|^{2} \\
& \left.-3\left|\frac{W(S, T)}{m_{11}^{3}}\right|^{2}\right\}
\end{aligned}
$$

Using the expression for $g_{h}$ in terms of $S$ and $T$, one can rewrite (29) in a more suggestive manner 


$$
\begin{aligned}
V_{\text {eff }} & =m_{11}^{4} e^{-3(\sigma+\gamma)}\left\{\left|c_{1}+\left(1+\frac{3}{2 b_{0} g_{h}^{2}}\right) \frac{\tilde{W}(S, T)}{m_{11}^{3}}\right|^{2}\right. \\
& \left.-\left[6 c_{3}^{2} \frac{(4 \pi)^{2 / 3}}{b_{0}^{2} g_{h}^{2}}(T+\bar{T})+12 c_{3} \frac{(4 \pi)^{2 / 3}}{b_{0}^{2}}(S+\bar{S})(T+\bar{T})\right] \frac{|\tilde{W}|^{2}}{m_{11}^{6}}\right\}
\end{aligned}
$$

At this point it is interesting to compare the form of $V_{\text {eff }}$ in (30) with the one obtained in compactifications of the perturbative heterotic string with hidden sector gaugino condensation and $H_{i j k}$ field strength turned on [5]. Here, in the simplest scenario, there are also two moduli $S$ and $T$ related to the dilaton $\phi$ and scale factor $e^{\sigma}$ via $\operatorname{Re} S=e^{3 \sigma} \phi^{-3 / 4}$ and $\operatorname{Re} T=e^{\sigma} \phi^{3 / 4}$. The relevant superpotential has the same form as in (25) except that the term in $T$ in the exponential is absent. One finds an effective potential $V_{e f f}^{(p)}$ given by

$$
V_{\text {eff }}^{(p)}=\frac{1}{16} M_{p l}^{4} e^{-6 \sigma} \phi^{-3 / 2}\left|c+h\left[\left(\frac{3}{2 b_{0}}\right)(S+\bar{S})+1\right] e^{-\frac{3 S}{2 b_{0}}}\right|^{2}
$$

This potential reproduces the perfect square form obtained explicitly from compactification only up to the power law correction term proportional to $(S+\bar{S})$ inside [ ] in (31). In this theory, the four dimensional gauge coupling $g^{-2}=\operatorname{Re} S$, so such a correction is of order $3 /\left(b_{0} g^{2}\right)$.

Now we return to the $V_{\text {eff }}$ in the M-theory case. It is clear that there are two corrections terms present, proportional to $1 / g_{h}^{2}$, but in addition there is a third term (the last term in (30) ) which is not a correction in this sense, and which represents a deviation away from a perfect square form of the potential. This is a direct consequence of having both an $S$ and $T$ dependence in the superpotential. Thus we should in principal find such terms in our naive compactification effective potential in (20), along with perfect square terms.

In fact this lack of perfect square structure fits in with our earlier observations concerning $V_{\text {eff }}$ in (20). To try and match (30) with (20), let us consider the more likely possibility discussed earlier, namely $G_{a b c}=(4 \pi)^{5 / 3} c m_{11}^{3} \epsilon_{a b c} \delta\left(x^{11}-\pi \rho\right)$, and $\operatorname{Tr}\left\langle\bar{\chi}_{(4)} \Gamma_{a b c} \chi_{(4)}\right\rangle=$ $(4 \pi)^{5 / 3} h \mu^{3} \epsilon_{a b c}$, with $c$ real and $h$ complex. Then only the integrals $B_{2} \ldots B_{4}$ are relevant in (20). The moduli independent parts of these integrals then reproduce the perfect square term in (30) with $\left|c_{1}\right|^{2}=c^{2} m_{11}^{6} \int_{X_{2}} d^{6} x\left(\sqrt{g^{(0)}} \epsilon^{2}\right)$ and $\left|c_{2}\right|^{2}=|h|^{2} m_{11}^{6} \int_{X_{2}} d^{6} x\left(\sqrt{g^{(0)}} \epsilon^{2}\right)$. One can expect that $m_{11}^{6} \int_{X_{2}} d^{6} x\left(\sqrt{g^{(0)}} \epsilon^{2}\right)$ will be of order 1 since it involves the metric $g_{i j}^{(0)}$.

However, there are additional moduli dependent pieces to $B_{2} \ldots B_{4}$, which are proportional to $e^{\gamma}$. Interestingly, the additional factors $(S+\bar{S})(T+\bar{T})$ found $(30)$ have also this $\gamma$ 
dependence, but there are difficulties. Firstly the additional terms in (30) are proportional to $|\tilde{W}|^{2}$ and not $|W|^{2}$ as implied by (20). Secondly the coefficients do not match up precisely, (e.g. the factors of $b_{0}$ in (30) are absent in the integrals $B_{2} \ldots B_{4}$.) Nevertheless the coefficients do resemble each other in that one has $\int_{X_{2}} d^{6} x \sqrt{g^{(0)}} \alpha\left(g^{(0)}, F\right)$ in $c_{3}$ and $\int_{X_{2}} d^{6} x \sqrt{g^{(0)}} \epsilon^{2} \alpha\left(g^{(0)}, F\right)$ occuring in the second terms of $B_{2} \ldots B_{4}$ (23).

Clearly these difficulties are related to those raised earlier in connection with having tree level gage kinetic functions that are dependent on both $S$ and $T$. Certainly the troublesome moduli dependent factors in $B_{2} \ldots B_{4}$ are a consequence of the same phenomenon that lead to a tree level dependence on $T$ in the hidden sector gauge coupling.

One should add that the situation is somewhat worse if we entertain the possibility of turning on the Chern-Simons forms in $G_{11 a b c}$ at each of the two $Z_{2}$ fixed points. In such an event, it does not seem likely that $B_{1} \ldots B_{4}$ have the structure necessary to reproduce any perfect square terms. It is hard to be definitive because in this case it is difficult to obtain an explicit dependence of these integrals on the coordinate $x^{11}$. This is because the terms we are turning on in $G_{11 a b c}$ are not covariantly constant so the analysis that lead to (23) is not obviously applicable.

Putting all these various problems aside, we can at least try to make some preliminary observations concerning the possible stabilization of the moduli expectation values. One of the motivations in trying to derive an effective potential from having both $G_{11 a b c}$ and $\langle\bar{\chi} \chi\rangle$ non vanishing was to hopefully avoid the usual runaway problem concerning the values of $S$ and $T$ at the minimum of $V_{\text {eff }}$. Banks and Dine [10 have already discussed the situation in the context of M-theory, at least for superpotentials of the form (25) but with $c_{1}=0$, i.e. they do not consider the more general situation of allowing nonvanishing $G_{11 a b c}$. Translating this to our potential (20), it appears possible that one might achieve stabilization of $\sigma$ and $\gamma$ (and hence the radius $\rho$ ) if one turns on e.g. $G_{11 a b c}$ at the boundary $X_{2}$ only and proportional to $\epsilon_{a b c}$, which was the first scenario discussed earlier. Although we do not present details here, what appears to be important in possibly achieving this stabilization is the relative minus signs of the moduli dependent parts of $B_{2} \ldots B_{4}$ compared to the moduli independent parts.

Finally, before ending this section, we comment on the results we have presented here, and the effective superpotentials obtained by [8] when applying the Scherk-Schwarz compactification procedure in the context of M-theory defined on $M_{4} \times X \times S^{1} / Z_{2}$. Such a comparison is motivated by the ideas presented in [7], that the Scherk-Schwarz mechanism applied in this way is effectively equivalent to gaugino condensation. One of the key features of the induced superpotentials in [8] was that the axionic shift symmetry 
associated with the modulus $S$ is violated. We have found no evidence of this from the form of the effective potential we have obtained in 20, 23 ). Furthermore if one added a linear term in $S$ to the superpotential (25), (to simulate the term found in [8] ), there are additional terms, some of which will violate $S$ shift symmetry in the corresponding scalar potential that again we have no evidence for in (20,23).

\section{Soft terms}

In this section we make some preliminary comments on the pattern of soft masses in the observable sector. At tree level in the observable sector the no-scale structure appears which is well known already from the weakly coupled heterotic string models. Since the observable superpotential is of no-scale type (trilinear terms in matter fields) the dependence of the scalar potential on matter fields $C$ relevant for the calculation of masses is of the form

$$
V=V\left(T+\bar{T}-|C|^{2}\right)
$$

which implies

$$
\left.\frac{\partial^{2} V}{\partial_{C} \partial_{\bar{C}}}\right|_{m i n, C=0}=-\left.\frac{\partial V}{\partial T}\right|_{m i n, C=0}=0
$$

However, there is no exact symmetry of the full Lagrangian which could prevent scalar masses from arising through radiative corrections (supersymmetry is broken and massive gravitino couples to observable fields). Similarly, gaugino masses will be generated through radiative corrections. In this respect the situation is similar to that in no-scale models studied in the past [12]. However, one can speculate that in some sense gaugino masses can arise even at tree level. The inverse gauge coupling can be regarded as the real part of the $x^{11}$-dependent holomorphic function $f$ of $S$ and $T$ superfields

$$
f=\alpha_{1} S+\beta_{1} T-T \Delta\left(x^{11}\right)
$$

where $\alpha_{1}$ and $\beta_{1}$ are constants ( $\beta_{1}$ is immediately given by (13) ) and $\Delta\left(x^{11}=0\right)=0$. In reality one expects that in quantum M-theory the singularities associated with the zero-thickness of the boundary are regularized by thickening the boundaries, the effective thickness being of the order of $m_{11}^{-1}$, as discussed earlier. In this case the resulting $\Delta_{e f f}(0) \approx \Delta\left(m_{11}^{-1}\right)$ can be different from zero. Now, in 4 d supergravity the tree-level gaugino masses are proportional to $f_{s} F^{S}+f_{T} F^{T}$. One doesn't know at this point the relation of the $F^{S, T}$-terms at $x^{11}=0$ to those which we can compute at the strongly 
coupled boundary using the effective Lagrangian derived here, but these quantities computed at the strongly coupled boundary in general don't have to both vanish. Of course, one can say that there exist M-theory models which are similar to weakly coupled string models, in these models one would expect in analogy T-dependent threshold corrections and future interesting structure in "twisted" sectors, but in this paper we want to restrict ourselves to the specific model at hand and to separate facts from speculations.

\section{Conclusions}

In this paper we have derived the explicit form, and discussed properties, of moduli dependent effective potentials as arising from compactification of M-theory when one of the boundaries supports a strongly interacting gauge sector and induces gaugino condensation. Consideration has been given to allowing non vanishing components of the three form field strength $G_{11 A B C}$. We have found that the naive process of trying to match the potential of moduli obtained by compactifying terms in the M- theory action, with the potential obtained from the superpotential $W(S, T)$ given in (30) is problematic. The origin of these difficulties appears to be due to the 'tree level' dependence of the hidden sector gauge coupling on the moduli $S$ and $T$. As we have argued in the section 3, although one may try and make sense of this by arguing that the $S$ and $T$ dependence might be from different orders in perturbation theory, this is at odds with what one obtains from compactification. Such difficulties are not apparent when the same ideas are employed in the perturbative heterotic string. It could be that these problems are an indication that in considering gaugino condensation in M-theory, and not carefully integrating out massive modes in obtaining an effective theory at scales $\ll m_{11}$, (rather using a more naive approach which worked in the perturbative case),leads to difficulties. Perhaps a related point is to understand deeper issues concerning the effective four dimensional theory obtained from M-theory for example the connection between Wilsonian and physical gauge couplings, which has revealed many subtle issues in the past [13] (and references therein ).

\section{Acknowledgments}

The work of Z.L. was partially supported by Polish Commitee for Scientific Research grant 2 P03B 040 12, and by M.Curie-Sklodowska Foundation and Polish-French Collaborative Projects Programme. The work of S.T. was supported by the Royal Society of Great 
Britain.

\section{References}

[1] J. Polchinski, String Duality, eprint hep-th/9607050.

[2] E. Witten, Nucl. Phys. B 471 (1996) 135.

[3] P. Horava, E. Witten, Nucl. Phys. B 475 (1996) 94; P. Horava, E. Witten, Nucl. Phys. B 475 (1996) 94.

[4] J.P. Derendinger, L.E. Ibáñez, H.P. Nilles, Phys.Lett. 155B (1985) 467; ; T.R. Taylor, Phys.Lett. 164B (1985) 43.

[5] M. Dine, R. Rohm, N. Seiberg, E. Witten, Phys.Lett. 156B (1985) 55.

[6] P. Horava, Phys. Rev. D 54 (1996) 7561.

[7] I. Antoniadis, M. Quiros, Phys. Lett. B392 (1997) 61; hep-th/9705037 (Nucl. Phys. B to appear; preprint CERN-TH/97-165, CPTH-S548.0797, IEM-FT-160/97 , eprint hep-th/9707208.

[8] E. Dudas, C. Grojean, preprint CERN-TH/97-79, LPTHE-ORSAY 97/17 Saclay T97/038, eprint hep-th/9704177.

[9] T. Li, J.L. Lopez and D.V. Nanopoulos, hep-ph/9702237; hep-ph/9704247.

[10] T. Banks and M. Dine Nucl.Phys. B479 (1996), 173.

[11] S. Ferrara, L. Girardello, H.P. Nilles, Phys.Lett. 125B (1983) 457.

[12] J. Ellis, D.V. Nanopoulos, M. Quiros and F. Zwirner, Phys. Lett. B180 (1986) 83; J. Ellis, A.B. Lahanas, D.V. Nanopoulos, M. Quiros and F. Zwirner, Phys. Lett. B188 (1987) 408.

[13] V. Kaplunovsky and J. Louis, Nucl.Phys. B422 (1994), 57; Nucl.Phys. B444 (1995), 191.

[14] H.P. Nilles and S. Stieberger, Nucl.Phys. B499 (1997), 3.

[15] H.P. Nilles, M. Olechowski and M. Yamaguchi, preprint TUM-HEP-282/97, SFB$375 / 201$, eprint hep-th/9707143. 
[16] A. Lukas, B.A. Ovrut and D. Waldram, preprint UPR-771T, PUPT-1723, HUBEP-97/51, hep-th/9710208. 